\title{
LAJOS TAKÁCS
}

\section{A queuing model with feedback}

Revue française d'automatique, d'informatique et de recherche opérationnelle. Recherche opérationnelle, tome 11, no 4 (1977), p. 345-354.

<http://www.numdam.org/item?id=RO_1977_11_4_345_0>

(C) AFCET, 1977, tous droits réservés.

L'accès aux archives de la revue « Revue française d'automatique, d'informatique et de recherche opérationnelle. Recherche opérationnelle » implique l'accord avec les conditions générales d'utilisation (http://www.numdam.org/ legal.php). Toute utilisation commerciale ou impression systématique est constitutive d'une infraction pénale. Toute copie ou impression de ce fichier doit contenir la présente mention de copyright.

\section{Numdam}

Article numérisé dans le cadre du programme

Numérisation de documents anciens mathématiques

http://www.numdam.org/ 


\title{
A QUEUING MODEL WITH FEEDBACK $\left({ }^{*}\right)$
}

\author{
by Lajos TAKÁCS ( ${ }^{1}$ )
}

\begin{abstract}
In the time interval $(0, \infty)$ customers arrive at a service system in accordance with $u$ Poisson process and form a queue in a waiting room. The customers are served by a single server in a service room in order of arrival. The service times are mutually independent random variables having $a$ common exponential distribution. After each service a customer may return to the waiting room with a constant probability. Every time the service room becomes empty all the customers in the waiting room and additional $r$ customers $(r \geqq 1)$ enter the service room. In this paper the distributions of the queue size, the waiting time and the total time spent in the system by a customer are determined for a stationary process.
\end{abstract}

\section{INTRODUCTION}

The object of this paper is to find the limit distributions (stationary distributions) of the queue size, the waiting time and the total time spent in the system by a customer for a single-server queue with feedback. It is assumed that in the time interval $(0, \infty)$ customers arrive at a service system in accordance with a Poisson process of density $\lambda$ and form a queue in a waiting room. The customers are served by a single server in a service room in order of arrival. The service times are mutually independent random variables having the same distribution function

$$
H(x)= \begin{cases}1-e^{-\mu x} & \text { for } x \geqq 0, \\ 0 & \text { for } x<0,\end{cases}
$$

and are independent of the arrival times. After each service a customer may return to the waiting room with probability $p$ where $0 \leqq p<1$ or may depart permanently with probability $q=1--p$. Every time the service room becomes empty, all the customers in the waiting room and additional $r$ customers $(r \geqq 1)$ enter the service room.

Denote by $\xi_{1}(t)$ the number of customers in the waiting room and by $\xi_{2}(t)$ the number of customers in the service room at time $t$. The possible values of $\xi_{1}(t)$ are $0,1,2, \ldots$ and the possible values of $\xi_{2}(t)$ are $1,2, \ldots$ We assume that the arrival times, the service times and the events of returns are independent of $\xi_{1}(0)$ and $\xi_{2}(0)$.

$\left({ }^{*}\right)$ Reçu décembre 1976.

(') Case Western Reserve University, Cleveland, Department of Mathematics and Statistics.

R.A.I.R.O. Recherche opérationnelle/Operations Research, vol. 11, $\mathrm{n}^{\circ}$ 4, nov. 1977 
In the particular case when $p=0$, that is, when there is no feedback the aforementioned model is equivalent to a counter model introduced by $D$. G. Lampard [1]. See also R. M. Phatarfod [2], [3], and the author [5].

\section{THE LIMIT DISTRIBUTION OF THE QUEUE SIZE}

We have the following result.

THEOREM $1:$ If $\lambda<\mu q$, then the limit distribution

$$
\lim _{t \rightarrow \infty} \mathbf{P}\left\{\xi_{1}(t)=i, \xi_{2}(t)=j\right\}=P_{i j}
$$

exists for $i \geqq 0$ and $j \geqq 1$ and is independent of the joint distribution of $\xi_{1}(0)$ and $\xi_{2}(0)$. The generating function

$$
P(w, z)=\sum_{i=0}^{\infty} \sum_{j=1}^{\infty} P_{i j} w^{i} z^{j}
$$

is given by

$$
P(w, z)=\frac{(\mu q-\lambda) z\left\{[Q(w)]^{r}-[z Q(z)]^{r}\right\}}{r[\mu q+\mu p w-(\lambda+\mu) z+\lambda w z]}
$$

for $|w| \leqq 1$ and $|z| \leqq 1$ where

$$
Q(z)=\sum_{n=0}^{\infty} A_{n}(\lambda z-\lambda)^{n}(\lambda z-\mu q)^{-n},
$$

$A_{0}=1$ and

$$
A_{n}=A_{n-1}\left[p+\frac{\lambda}{\mu}-\frac{\mu q}{\lambda}\left(p+\frac{\lambda}{\mu}\right)^{n}\right]\left[1-\left(p+\frac{\lambda}{\dot{\mu}}\right)^{n}\right]^{-1}
$$

for $n \geqq 1$. If $\lambda \geqq \mu q$, then $\lim _{t \rightarrow \infty} \mathbf{P}\left\{\xi_{1}(t)=i, \xi_{2}(t)=j\right\}=0$ regardless of the joint distribution of $\xi_{1}(0)$ and $\xi_{2}(0)$.

Proof: The random variables $\left\{\xi_{1}(t), \xi_{2}(t)\right\}$ form a homogeneous and irreducible Markov process with state spăce

$$
\{(i, j): i=0,1,2, \ldots, j=1,2, \ldots\} .
$$

If the process $\left\{\xi_{1}(t), \xi_{2}(t) \cdot\right\}$ has a stationary distribution, then the limit distribution (2) exists and is identical with the stationary distribution. If $\left\{\xi_{1}(t), \xi_{2}(t)\right\}$ has no stationary distribution, then

$$
\lim _{t \rightarrow \infty} \mathbf{P}\left\{\xi_{1}(t)=i, \xi_{2}(t)=j\right\}=0
$$

for all $i \geqq 0$ and $j \geqq 1$. 
If we suppose that $\left\{P_{i j}\right\}$ is a stationary distribution, then it satisfies the following system of equations

$$
(\lambda+\mu) P_{i j}=\lambda P_{i-1, j}+\mu p P_{i-1, j+1}+\mu q P_{i, j+1}
$$

for $i \geqq 1, j \geqq 1$,

$$
(\lambda+\mu) P_{0 j}=\mu q P_{0, j+1}
$$

for $1 \leqq j<r$,

$$
\begin{gathered}
(\lambda+\mu) P_{0 r}=\mu q P_{01}+\mu q P_{0, r+1} \\
(\lambda+\mu) P_{0, j+r}=\mu p P_{j-1,1}+\mu q P_{j, 1}+\mu q P_{0, j+r+1}
\end{gathered}
$$

for $j \geqq 1$ and

$$
\sum_{i=0}^{\infty} \sum_{j=1}^{\infty} P_{i j}=1
$$

Define $P(w, z)$ by (3). By (7), (8), (9), and (10) we obtain that

$$
P(w, z)=\frac{\mu z\left[(q+p w) G(w)-(q+p z) z^{r} G(z)\right]}{\mu q-(\lambda+\mu) z+(\lambda z+\mu p) w}
$$

for $|w| \leqq 1$ and $|z| \leqq 1$ where

$$
G(w)=\sum_{i=0}^{\infty} P_{i 1} w^{i}
$$

for $|w| \leqq 1$

If a stationary distribution $\left\{P_{i j}\right\}$ exists, then by $(11)$ we have $P(1,1)=1$, and (12) implies that

$$
\lim _{z \rightarrow 1} P(1, z)=(p+r) G(1)+G^{\prime}(1)=1
$$

and

$$
\lim _{w \rightarrow 1} P(w, 1)=\frac{\mu p G(1)+\mu G^{\prime}(1)}{\lambda+\mu p}=1 .
$$

Hence we get

$$
G(1)=\frac{\mu q-\lambda}{\mu r} .
$$

This shows at once that a stationary distribution cannot exist if $\lambda \geqq \mu q$. If $\lambda<\mu q$, then a stationary distribution exists and its generating function is given by (12) where $G(w)$ is still to be determined. In (12) we can determine $G(w)$ for $|w| \leqq 1$ by the requirement that $|P(w, z)| \leqq 1$ for $|w| \leqq 1$ and $|z| \leqq 1$. If $|w| \leqq 1$, then the denominator of (12) has a zero

$$
z=\frac{\mu(q+p w)}{\mu+\lambda(1-w)}
$$

vol. $11, \mathrm{n}^{\circ} 4$, nov. 1977 
in the unit circle $|z| \leqq 1$. If $z$ is equal to (17), then the numerator of (12) should vanish too, that is,

$$
(q+p w) G(w)=(q+p z) z^{r} G(z)
$$

whenever $z$ is defined by (17) and $|w| \leqq 1$.

Let

$$
R(w)=(q+p w) G(w) / G(1)
$$

for $|w| \leqq 1$. Then $R(1)=1$ and by (18) we have

$$
R(w)=z^{r} R(z)
$$

whenever $z$ is defined by (17) and $|w| \leqq 1$. Hence it follows that

$$
R(w)=[Q(w)]^{r}
$$

where $Q(1)=1$,

and

$$
Q(w)=U(w) Q(U(w))
$$

$$
U(w)=\frac{\mu(q+p w)}{\mu+\lambda(1-w)}
$$

for $|w| \leqq 1$.

By (12), (19) and (21) we get (4) where only $Q(w)$ remains to be determined. If we define $U_{1}(w)=U(w)$ and $U_{n+1}(w)=U\left(U_{n}(w)\right)$ for $n=1,2, \ldots$, then by (22) it follows that

$$
Q(w)=\prod_{n=1}^{\infty} U_{n}(w)
$$

for $|w| \leqq 1$. We can easily' see that

$$
U_{n}(w)=\frac{\alpha_{n} w+\beta_{n}}{\gamma_{n} w+\delta_{n}}
$$

for $n=1,2, \ldots$ where

$$
\left\|\begin{array}{ll}
\alpha_{n} & \beta_{n} \\
\gamma_{n} & \delta_{n}
\end{array}\right\|=C_{n}\left\|\begin{array}{cc}
\mu p & \mu q \\
-\lambda & \mu+\lambda
\end{array}\right\|^{n}
$$

and $C_{n} \neq 0$. If we choose $C_{n}=\mu q-\lambda$, then we have

$$
\begin{aligned}
& \alpha_{n}=\mu q(\lambda+\mu p)^{n}-\lambda \mu^{n}, \beta_{n}=\mu q\left[\mu^{n}-(\lambda+\mu p)^{n}\right] \\
& \gamma_{n}=\lambda\left[(\lambda+\mu p)^{n}-\mu^{n}\right], \delta_{n}=\mu q \mu^{n}-\lambda(\lambda+\mu p)^{n} .
\end{aligned}
$$

However, we can also determine $Q(w)$ in a simpler way. Let us write

$$
Q(w)=A\left(\frac{\lambda w-\lambda}{\lambda w-\mu q}\right)
$$


for $|w| \leqq 1$. Then by (22) we get

$$
A(z)\left[1-\left(p+\frac{\lambda}{\mu}\right) z\right]=A\left(p z+\frac{\lambda z}{\mu}\right)\left[1-\frac{\mu q}{\lambda}\left(p+\frac{\lambda}{\mu}\right) z\right]
$$

and $A(0)=Q(1)=1$ : By expanding $A(z)$ into a Taylor series at $z=0$,

$$
A(z)=\sum_{n=0}^{\infty} A_{n} z^{n}
$$

the coefficients $A_{n}(n=0,1,2, \ldots)$ can be determined by (29). If we form the coefficient of $z^{n}$ in (29), then we get

$$
A_{n}-\left(p+\frac{\lambda}{\mu}\right) A_{n-1}=\left(A_{n}-\frac{\mu q}{\lambda} A_{n-1}\right)\left(p+\frac{\lambda}{\mu}\right)^{n}
$$

for $n=1,2, \ldots$ and $A_{0}=1$. This proves formulas (5) and (6).

\section{THE LIMIT DISTRIBUTION OF THE WAITING TIME}

Denote by $\xi_{n}$ the queue size, that is, the number of customers in the system, immediately before the $n$-th customer arrives. We can easily see that if $\lambda<\mu q$, then the limit distribution

$$
\lim _{n \rightarrow \infty} \mathbf{P}\left\{\xi_{n}=j\right\}=P_{j}
$$

exists for $j=1,2, \ldots$ and is independent of the joint distribution of $\xi_{1}(0)$ and $\xi_{2}(0)$. If $\lambda \geqq \mu q$, then $\lim _{n \rightarrow \infty} \mathbf{P}\left\{\dot{\xi}_{n}=j\right\}=0$ for $j=1,2, \ldots$

Il $\lambda<\mu q$, then the generating function

$$
\Phi(z)=\sum_{j=1}^{\infty} P_{j} z^{j}
$$

is given by

$$
\Phi(z)=P(z, z)=[Q(z)]^{r}\left(\frac{1-z^{r}}{r-r z}\right)\left(\frac{\mu q z-\lambda z}{\mu q-\lambda z}\right)
$$

for $|z| \leqq 1$ where $Q(z)$ is defined by (5) and (6).

Denote by $\eta_{n}$ the waiting time of the $n$-th customer.

THEOREM $2:$ If $\lambda<\mu q$, then the limiting distribution

$$
\lim _{n \rightarrow \infty} \mathbf{P} .\left\{\eta_{n} \leqq x\right\}=W(x)
$$

exists and is independent of the joint distribution of $\xi_{1}(0)$ and $\xi_{2}(0)$. The LaplaceStieltjes transform

$$
\Omega(s)=\int_{0}^{\infty} e^{-s x} d W(x)
$$

vol. $11, \mathrm{n}^{\circ} 4$, nov. 1977 
is given by

$$
\Omega(s)=\Phi\left(\frac{\mu}{\mu+s}\right)
$$

for $\operatorname{Re}(s) \geqq 0 \quad$ where $\Phi(z)$ is defined by (34). If $\lambda \geqq \mu q$, then $\lim _{n \rightarrow \infty} \mathbf{P}\left\{\eta_{n} \leqq x\right\}=0$ for all $x$.

Proof: Since $\eta_{n}$ can be represented as a sum of $\xi_{n}$ independent and identically distributed random variables with distribution function (1), (35) and (37) follow immediately from (32) and (33).

Define

$$
M_{v}=\sum_{j=1}^{\infty} j^{v} P_{j}
$$

for $v=1,2, \ldots$ and write $M=M_{1}$ and $D=M_{2}-M_{1}^{2}$.

THEOREM $3:$ If $\lambda<\mu q$, then

$$
M=\frac{r(\lambda+\mu p)}{\mu q-\lambda}+\frac{r+1}{2}+\frac{\lambda}{\mu q-\lambda}
$$

and

$$
D=\frac{r \mu(\lambda+\mu q)(\lambda+\mu p)}{(\mu q-\lambda)^{2}(\lambda+\mu+\mu p)}+\frac{r^{2}-1}{12}+\frac{\lambda \mu q}{(\mu q-\lambda)^{2}} .
$$

Proof: By (34) we obtain that

$$
M=\Phi^{\prime}(1)=r Q^{\prime}(1)+\frac{r+1}{2}+\frac{\lambda}{\mu q-\lambda}
$$

and

$$
\begin{aligned}
D & =\Phi^{\prime \prime}(1)+\Phi^{\prime}(1)-\left[\Phi^{\prime}(1)\right]^{2} \\
& =r\left[Q^{\prime \prime}(1)+Q^{\prime}(1)-\left(Q^{\prime}(1)\right)^{2}\right]+\frac{r^{2}-1}{12}+\frac{\lambda \mu q}{(\mu q-\lambda)^{2}} .
\end{aligned}
$$

Il we form the derivatives of (22) at $w=1$, then we get

$$
Q^{\prime}(1)=\frac{U^{\prime}(1)}{1-U^{\prime}(1)}
$$

and

$$
Q^{\prime \prime}(1)+Q^{\prime}(1)-\left[Q^{\prime}(1)\right]^{2}=\frac{U^{\prime \prime}(1)+U^{\prime}(1)-\left[U^{\prime}(1)\right]^{2}}{\left[1-U^{\prime}(1)\right]^{2}\left[1+U^{\prime}(1)\right]} .
$$

Since $U^{\prime}(1)=(\lambda+\mu p) / \mu$ and $U^{\prime \prime}(1)=2 \lambda(\lambda+\mu p) / \mu^{2}$ by the above formulas we get (39) and (40). 
From (37) it follows that if $\lambda<\mu q$, then

$$
\int_{0}^{\infty} x d W(x)=M / \mu
$$

and

$$
\int_{0}^{\infty}\left(x-\frac{M}{\mu}\right)^{2} d W(x)=(D+M) / \mu^{2} .
$$

\section{THE LIMIT DISTRIBUTION OF THE SOJOURN TIME}

Denote by $\theta_{n}$ the total time spent in the system by the $n$-th customer. Obviously, the distribution of $\theta_{n}$ depends solely on the distribution of $\xi_{n}$, the number of customers in the system immediately before the arrival of the $n$-th customer. If $\lambda<\mu q$, then $\xi_{n}$ has a limit distribution which does not depend on the initial distribution of the process. Consequently, if $\lambda<\mu q$, then $\theta_{n}$ also has a limit distribution which does not depend on the initial distribution of the process. The limit distribution of $\theta_{n}$ is evidently the same as the distribution of $\theta_{n}$ in the case where $\mathbf{P}\left\{\xi_{n}=j\right\}=P_{j}(j=1,2, \ldots)$ defined by (32). Thus we assume that $\lambda<\mu q$ and that $\mathbf{P}\left\{\xi_{n}=j\right\}=P_{j}(j=1,2, \ldots)$ is given by (33) and (34), and give a method of finding the distribution of $\theta_{n}$.

Denote by $\theta_{n}^{(k)}$ the total time spent in the system by the $n$-th customer until his $k$-th service ends if he joins the queue at least $k$ times. Denote by $\zeta_{n}^{(k)}$ the number of customers in the system immediately after the $k$-th service of the $n$-th customer ends. The $n$-th customer is not included in $\zeta_{n}^{(k)}$ even if he returns at least $k+1$ times. If $\xi_{n}$ has a stationary distribution, then the expectation

$$
\Phi_{k}(s, z)=\mathbf{E}\left\{e^{-s \theta_{n}^{(k)} z_{n}^{(k)}}\right\}
$$

exists for $\operatorname{Re}(s) \geqq 0$ and $|z| \leqq 1$ and is independent of $n$. We can easily see that

$$
\Phi_{k+1}(s, z)=\Phi_{k}\left(s, \frac{\mu(q+p z)}{\mu+s+\lambda(1-z)}\right) \frac{\mu z^{r}}{\mu+s+\lambda(1-z)}
$$

for $k=0,1,2, \ldots$ where $\Phi_{0}(s, z)=\Phi(z)$ is defined by (34). The proof of (48) follows the same lines as the proof of (28) in reference [4]. Define

$$
\Phi(s, z)=q \sum_{k=1}^{\infty} p^{k-1} \Phi_{k}(s, z)
$$

for $\operatorname{Re}(s) \geqq 0$ and $|z| \leqq 1$. The function $\Phi(s, z)$ is completely determined by the recurrence formula (48) and the initial condition $\Phi_{0}(s, z)=\Phi(z)$.

THEOREM $4:$ If $\lambda<\mu q$, then the limit distribution

$$
\lim _{n \rightarrow \infty} \mathbf{P}\left\{\theta_{n} \leqq x\right\}=K(x)
$$

vol. $11, \mathrm{n}^{\circ} 4$, nov. 1977 
exists and is independent of the initial distribution of the process. If $\operatorname{Re}(s) \geqq 0$, then

$$
\int_{0}^{\infty} e^{-s x} d K(x)=\Phi(s, 1)
$$

where $\Phi(s, z)$ is defined by (49).

Proof: The probability that the $n$-th customer joins the queue exactly $k$ times (including his original arrival) is $q p^{k-1}$ for $k=1,2, \ldots$ Thus we have

$$
\mathbf{E}\left\{e^{-s \theta_{n}}\right\}=q \sum_{k=1}^{\infty} p^{k-1} \mathbf{E}\left\{e^{-s \theta_{n}^{(k)}}\right\}
$$

for $\operatorname{Re}(s) \geqq 0$. If $\xi_{n}$ has a stationary distribution defined by (33), then (52) reduces to $\Phi(s, 1)$ and this proves $(51)$.

We note that in (49) we can express $\Phi_{k}(s, z)$ in an explicit form. Let us write

$$
U(s, z)=\frac{\mu(q+p z)}{\mu+s+\lambda(1-z)}
$$

and define $U_{n}(s, z)(n=0,1,2, \ldots)$ by the recurrence formula

$$
U_{n+1}(s, z)=U\left(s, U_{n}(s, z)\right)=U_{n}(s, U(s, z))
$$

for $n=0,1,2, \ldots$ where $U_{0}(s, z)=z$. It is easy to see that if we define

$$
\Phi_{k}(s, z)=\Phi\left(U_{k}(s, z)\right) \prod_{j=0}^{k-1}\left(\frac{\mu\left[U_{j}(s, z)\right]^{r}}{\mu+s+\lambda\left[1-U_{j}(s, z)\right]}\right)
$$

for $k=1,2, \ldots$, and if $\Phi_{0}(s, z)=\Phi(z)$, then (48) is satisfied for $k=0,1,2, \ldots$ In $(55)$ we have

$$
U_{k}(s, z)=\frac{\alpha_{k}(s) z+\beta_{k}(s)}{\gamma_{k}(s) z+\delta_{k}(s)}
$$

for $k=0,1,2, \ldots$ where

$$
\left\|\begin{array}{ll}
\alpha_{k}(s) & \beta_{k}(s) \\
\gamma_{k}(s) & \delta_{k}(s)
\end{array}\right\|=\left\|\begin{array}{cc}
\mu p & \mu q \\
-\lambda & \mu+\lambda+s
\end{array}\right\|^{k}
$$

From (48) it follows immediately that

$$
[\mu+s+\lambda(1-z)] \Phi(s, z)=\mu q z^{r} \Phi(z)+\mu p z^{r} \Phi\left(s, \frac{\mu(q+p z)}{\mu+s+\lambda(1-z)}\right)
$$

for $\operatorname{Re}(s) \geqq 0$ and $|z| \leqq 1$. By (58) we can easily determine the moments

$$
K_{m}=\int_{0}^{\infty} x^{m} d K(x)
$$

R.A.I.R.O. Recherche opérationnelle/Operations Research 
for $m=1,2, \ldots$ Define

$$
\Phi_{i j}=\frac{1}{i ! j !}\left(\frac{\partial^{i+j} \Phi(s, z)}{\partial s^{i} \partial z^{j}}\right)_{s=0, z=1}
$$

for $i=0,1,2, \ldots$ and $j=0,1,2, \ldots$ If we form the mixed partial derivatives of order $m$ of (58), then we obtain $m+1$ linear equations for the determination of $\Phi_{0 m}, \Phi_{1, m-1}, \ldots, \Phi_{m 0}$. These derivatives can be determined step by step for $m=1,2, \ldots$ and

$$
K_{m}=(-1)^{m} m ! \Phi_{m 0}
$$

yields the $m$-th moment of $K(x)$.

Introduce the notation

$$
a_{i j}=\frac{1}{i ! j !}\left[\frac{\partial^{i+j}}{\partial s^{i} \partial z^{j}}\left(\frac{\mu(q+p z)}{\mu+s+\lambda(1-z)}\right)\right]_{s=0, z=1}
$$

for $i \geqq 0$ and $j \geqq 0$. Then $a_{00}=1, a_{10}=-1 / \mu, a_{01}=(\lambda+\mu p) / \mu$, $a_{20}=1 / \mu^{2}, a_{11}=-(2 \lambda+\mu p) / \mu^{2}$ and $a_{02}=\lambda(\lambda+\mu p) / \mu^{2}$.

By (58) we obtain

$$
1+\mu \Phi_{10}=\mu p \Phi_{10}-p \Phi_{01}
$$

and

$$
\mu \Phi_{01}-\lambda=\mu r+\mu q \Phi^{\prime}(1)+(\lambda+\mu p) p \Phi_{01} .
$$

From (63)

$$
\Phi_{10}=-\left(1+p \Phi_{01}\right) / \mu q
$$

and from (64)

$$
\Phi_{01}=\left[\lambda+\mu r+\mu q \Phi^{\prime}(1)\right][\mu-(\lambda+\mu p) p]^{-1} .
$$

where $\Phi^{\prime}(1)=M$ is given by (39). Since $K_{1}=-\Phi_{10}$, by (65) and (66) we get

$$
K_{1}=\frac{1}{\mu q}+\frac{p}{\mu q}\left[\frac{2(\mu q-\lambda)(\lambda+\mu r)+\mu q \lambda(r+1)+\mu^{2} q(2 r-(r-1) q)}{2(\mu q-\lambda)[\mu-(\lambda+\mu p) p]}\right]
$$

If we form the partial derivatives of order two of (58), then we obtain the following equations for the determination of $K_{2}=2 \Phi_{20}$ :

$$
\begin{gathered}
\mu \Phi_{20}+\Phi_{10}=\mu p a_{20} \Phi_{01}+\mu p \Phi_{20}+\mu p a_{10} \Phi_{11}+\mu p a_{10}^{2} \Phi_{02}, \\
\Phi_{01}-\lambda \Phi_{10}+\mu \Phi_{11}= \\
=\mu p\left[a_{11} \Phi_{01}+a_{01} \Phi_{11}+2 a_{10} a_{01} \Phi_{02}+r \Phi_{10}+r a_{10} \Phi_{01}\right]
\end{gathered}
$$

vol. $11, \mathrm{n}^{\circ} 4$, nov. 1977 
and

$$
\begin{aligned}
& \mu \Phi_{02}-\lambda \Phi_{01}=\mu q\left[\frac{\Phi^{\prime \prime}(1)}{2}+r \Phi^{\prime}(1)+\left(\begin{array}{l}
r \\
2
\end{array}\right)\right] \\
&+\mu p\left[a_{02} \Phi_{01}+a_{01}^{2} \Phi_{02}+r a_{01} \Phi_{01}+\left(\begin{array}{l}
r \\
2
\end{array}\right)\right] .
\end{aligned}
$$

Now $\Phi_{02}$ can be determined by (70), $\Phi_{11}$ by (69) and finally $\Phi_{20}$ by (68).

\section{AN EXAMPLE}

Let us suppose that in the queuing process, $p=1 / 3$ and $\lambda / \mu=1 / 6$. Then $\lambda / \mu q=1 / 4$ and $\xi_{n}, \eta_{n}$ and $\theta_{n}$ have a limit distribution. The probabilities $\lim _{n \rightarrow x_{-}} \mathbf{P}\left\{\xi_{n}=j\right\}=P_{j}(j=1,2, \ldots)$ are determined by (34) where now

$$
Q(z)=3(z+2)(4-z)^{-2} .
$$

This follows from (5) where now $A_{0}=1, A_{1}=-3, A_{2}=2$ and $A_{n}=0$ for $n \geqq 3$. In this case (34) reduces to

$$
\Phi(z)=\left(\frac{1-z^{r}}{r(1-z)}\right)\left(\frac{3}{4-z}\right)\left(\frac{3(z+2)}{4-z}\right)^{r} .
$$

The Laplace-Stieltjes transform of the limiting distribution of $\eta_{n}$ is given by (37), and the Laplace-Stieltjes transform of the limiting distribution of $\theta_{n}$ is given by (51).

By (39) and (40) we obtain that

$$
M=(9 r+5) / 6
$$

and

By (67) we get

$$
D=\left(3 r^{2}+40 r+13\right) / 36 \text {. }
$$

$$
\mu K_{1}=(18 r+29) / 15
$$

and by (68), (69) and (70) it follows that

$$
\mu^{2} K_{2}=\left(16065 r^{2}+37025 r+23827\right) / 2475 \text {. }
$$

\section{REFERENCES}

1. D. G. Lampard, A Stochastic Process whose Successive Intervals between Events Form a First order Markov Chain. I. Journal of Applied Probability, 5 (1968), p. 648-668.

2. R. M. Phatarfod, Note on the Reversible Counters System of Lampard. Journal of Applied Probability, 11 (1974), p. 624-628.

3. R. M. Phatarfod, On the Reversible Counters System of Lampard. Journal of Applied Probability, 12 (1975), 639-646:

4. L. TAKÁCS, A Single-Server Queue with Feedback. Bell System Technical Journal, 42, 1963 , p. 505-519.

5. L. TAKÁCS, Some Remarks on a Counter Process. Journal of Applied Probability, 13, 1976, p. 623-627. 